\title{
Myocardial protective effect and transcriptome profiling of Naoxintong on cardiomyopathy in zebrafish
}

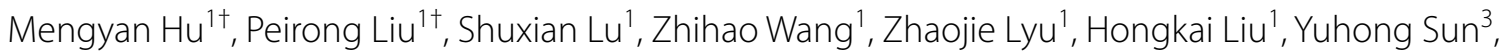
Feng Liu $^{3,4}$ and Jing $\operatorname{Tian}^{1,2^{*}}$

\begin{abstract}
Background: Cardiomyopathy is a kind of cardiovascular diseases, which makes it more difficult for the heart to pump blood to other parts of the body, eventually leading to heart failure. Naoxintong (NXT), as a traditional Chinese Medicine (TCM) preparation, is widely used in the treatment of cardiovascular diseases, including cardiomyopathy, while its underlying mechanism has not been fully elucidated. The purpose of this study is to investigate the therapeutic effect of NXT on cardiomyopathy and its molecular mechanism in zebrafish model.
\end{abstract}

Methods: The zebrafish cardiomyopathy model was established using terfenadine (TFD) and treated with NXT. The therapeutic effect of NXT on cardiomyopathy was evaluated by measuring the heart rate, the distance between the sinus venosus and bulbus arteriosus (SV-BA), the pericardial area, and the blood flow velocity of zebrafish. Then, the zebrafish hearts were isolated and collected; transcriptome analysis of NXT on cardiomyopathy was investigated. Moreover, the heg1 mutant of zebrafish congenital cardiomyopathy model was used to further validate the therapeutic effect of NXT on cardiomyopathy. Additionally, UPLC analysis combined with the zebrafish model investigation was performed to identify the bioactive components of NXT.

Results: In the TFD-induced zebrafish cardiomyopathy model, NXT treatment could significantly restore the cardiovascular malformations caused by cardiac dysfunction. Transcriptome and bioinformatics analyses of the TFD and TFD + NXT treated zebrafish developing hearts revealed that the differentially expressed genes were highly enriched in biological processes such as cardiac muscle contraction and heart development. As a cardiac development protein associated with cardiomyopathy, HEG1 had been identified as one of the important targets of NXT in the treatment of cardiomyopathy. The cardiovascular abnormalities of zebrafish heg1 mutant could be recovered significantly from NXT treatment, including the expanded atrial cavity and blood stagnation. QRT-PCR analysis further showed that NXT could restore cardiomyopathy phenotype in zebrafish through HEG1-CCM signaling. Among the seven components identified in NXT, paeoniflorin (PF) and salvianolic acid B (Sal B) were considered to be the main bioactive ones with myocardial protection.

Conclusion: NXT presented myocardial protective effect and could restore myocardial injury and cardiac dysfunction in zebrafish; the action mechanism was involved in HEG1-CCM signaling.

\footnotetext{
*Correspondence: tianjing@nwu.edu.cn

${ }^{\dagger}$ Mengyan Hu and Peirong Liu contributed equally to this work

${ }^{1}$ Western China Zebrafish Research Center for Human Diseases

and Drug Screening, The College of Life Sciences, Northwest University,

Xi'an 710069, China

Full list of author information is available at the end of the article
}

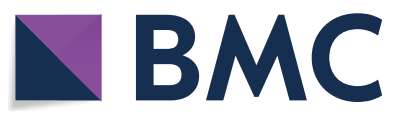

2021. Open Access This article is licensed under a Creative Commons Attribution 4.0 International License, which permits use, sharing, adaptation, distribution and reproduction in any medium or format, as long as you give appropriate credit to the original author(s) and the source, provide a link to the Creative Commons licence, and indicate if changes were made. The images or other third party material in this article are included in the article's Creative Commons licence, unless indicated otherwise in a credit line to the material. If material is not included in the article's Creative Commons licence and your intended use is not permitted by statutory regulation or exceeds the permitted use, you will need to obtain permission directly from the copyright holder. To view a copy of this licence, visit http://creativecommons.org/licenses/by/4.0/. The Creative Commons Public Domain Dedication waiver (http://creativeco mmons.org/publicdomain/zero/1.0/) applies to the data made available in this article, unless otherwise stated in a credit line to the data. 
Keywords: Cardiomyopathy, Naoxintong (NXT), Zebrafish, Transcriptome, HEG1

\section{Background}

Cardiomyopathy, a kind of cardiovascular diseases (CVD), is a mechanical and cardiac dysfunction of the heart muscle, which can lead to cardiac death or heart failure [1]. The most typical feature of cardiomyopathy is abnormal myocardial structure and systolic function. Because the regulation of myocardial contractility directly affects the myocardium and the general cardiovascular system, cardiomyopathy is often accompanied by ventricular enlargement caused by intraventricular blood stagnation, resulting in cardiac pumping dysfunction [2]. Cardiomyopathies are classified according to phenotype as hypertrophic cardiomyopathy (HCM), dilated cardiomyopathy (DCM), restrictive cardiomyopathy (RCM), arrhythmogenic right ventricular cardiomyopathy (ARVC), and unclassified cardiomyopathies [3]. Among them, DCM, characterized by cardiac enlargement and loss of systolic function, has the highest prevalence among all cardiomyopathies [4].

Zebrafish (Danio rerio) has become a powerful tool for the study of human diseases. Some or most human pathological phenomena can be observed in zebrafish. The heart is the first functioning organ to be formed during zebrafish embryogenesis [5, 6]. Although the structure of the zebrafish embryonic heart is simpler than that of mammals, with only two chambers, atrium and ventricle, the morphological processes and the regulatory molecular pathways of the zebrafish heart formation are similar to those of mammalians. Zebrafish embryos and larvae are small and transparent, and can be used to observed organ formation and related biological processes, especially cardiovascular development, with high resolution in vivo. Furthermore, with the benefit from the transgenic lines and genetic manipulation tools available such as CRISPRCas9 technology [7], zebrafish has become particularly useful in cardiogenesis. There are several zebrafish disease models generated and applied not only for the research of related diseases, but also for the related drug screening and therapeutic efficacy analysis [8]. $\mathrm{Gu}$ et al. developed a dilated cardiomyopathy model in zebrafish larvae with terfenadine (TFD). It is characterized by atria and ventricle swelling, decreased blood circulation and heart rate, which was a good model of non-ischemic heart failure [9]. In our previous study, by specific knocking out a heart development protein heg1 using CRISPR/Cas9 technology, we established a zebrafish congenital dilated cardiomyopathy model, heg1 ${ }^{\Delta 25}$ mutant. Zebrafish heg1 $1^{\Delta 25}$ mutant showed very obvious pericardial swelling, pericardial edema, and heart rate slowing, which were similar to those in patients with heart failure [10]. heg1 ${ }^{\Delta 25}$ mutant was also used to verify the therapeutic effect of Traditional Chinese Medicine (TCM) and monomers in the treatment of cardiovascular diseases [10].

In recent years, TCM has been more and more widely used in the treatment of cardiovascular diseases [11]. Naoxintong (NXT) is a well-known prescribed TCM, which is commonly used for the prevention and treatment of cardiovascular and cerebrovascular diseases. It has been clinically used for more than two decades [12]. NXT consists of 16 herbs, including Astragali Radix (Huangqi), Salviae miltiorrhizae Radix et Rhizoma (Danshen), Paeoniae Radix Rubra (Chishao), Angelicae Sinensis Radix (Danggui), Cinnamomi Ramulus (Guizhi), Persicae Semen (Taoren), Chuanxiong Rhizoma (Chuanxiong), Spatholobi Stem (Jixueteng), Mori Ramulus (Sangzhi), Carthami flos (Honghua), Achyranthis (Niuxi), Olibanum (Ruxaing), Myrrha Achyranthis (Moyao), Pheretima (Dilong), Scorpio (Quanxie), Hirudo (Shuizhi) [13]. Recent studies have shown that NXT plays a cardioprotective role by affecting inflammation, apoptosis, oxidative stress, neovascularization, insulin sensitivity and lipid/glucose metabolism, as well as restoring ischemia injury $[14,15]$. In the analysis of blood samples from 69 patients with type 2 diabetes mellitus (T2DM) treated with NXT, it was found that NXT was able to restore the effects of HDL on the proliferation, apoptosis and angiogenesis of human umbilical vein endothelial cells [16]. In the rat coronary microembolism (CME) model, NXT significantly reduced the number of CME and cardiomyocyte apoptosis [17]. In the mouse model of myocardial ischemia/reperfusion (I/R) injury, Wang et al. found that NXT improved ventricular function and reduced infarct size after injury by inhibiting NLRP3 inflammatory vesicle activation [18]. In $\mathrm{H}_{2} \mathrm{O}_{2}$-induced endothelial cell injury, NXT aqueous extract could reduce apoptosis and autophagy by inhibiting the activation of caspase-3/ PARP-1 signaling pathway [19]. In terms of vascular protection, NXT could improve ischemia-reperfusion injury as well as lipid metabolism, and also had the effect of inhibiting vascular inflammation and protecting the vascular endothelium [20]. Our previous study also revealed that NXT could repair cellular hypoxia and glucose damage in cell OGD/R model, and improve the symptoms of cardiac/vascular ischemia by regulating COX2-VEGF/ $\mathrm{NF} \kappa \mathrm{B}$ signaling in the zebrafish thrombosis model [15]. However, the underlying molecular mechanism of NXT 
in the treatment of myocardial injury has not been fully elucidated, and still need to be further studied.

In this study, TFD-induced and congenital zebrafish dilated cardiomyopathy models were applied to evaluate the myocardial protective effect of NXT on cardiomyopathy. The potential molecular mechanism of its action was investigated through transcriptome profiling analysis.

\section{Materials and methods}

\section{Ethics statement}

The zebrafish experiments were conducted according to the ethical guidelines of Northwest University. All experimental protocols were approved by the Experimental Animal Management and Ethics Committee of Northwest University, and the ethical code was NWU-AWC-20190103Z.

\section{Preparation of NXT extract}

Naoxintong Capsule (NXT) was kindly provided by Shaanxi Buchang Pharmaceuticals. The extraction process of NXT was carried out by water extraction. In brief, $10 \mathrm{~g}$ of NXT powder was soaked in $100 \mathrm{ml}$ distilled water for $30 \mathrm{~min}$, followed by ultrasonic $(250 \mathrm{~W}, 50 \mathrm{kHz})$ for $30 \mathrm{~min}$, and evaporated to $10 \mathrm{ml}$ and filtered; the stock concentration is $1 \mathrm{~g} / \mathrm{ml}$ and kept at $-80^{\circ} \mathrm{C}$.

\section{Zebrafish maintenance and embryos handling}

All adult zebrafish were maintained in zebrafish culture system with a light cycle of $14 \mathrm{~h}$ light/10 h dark. Zebrafish embryos were produced by natural crosses and cultured in egg water at $28.5{ }^{\circ} \mathrm{C}$ [21]. The following strains were used: $A B$ wild-type (wt) strain, transgenic $T g(c m l c 2: e G F P)$ strain expressing enhanced green fluorescent protein (GFP) in cardiomyocytes [22], $\mathrm{Tg}(f l k 1: e G F P)$ strain expressing enhanced green fluorescent protein (GFP) in blood vasculature [23], heg $1^{\Delta 25}$ mutant, $\operatorname{Tg}\left(\right.$ heg $1^{\Delta 25}$; cmlc2:eGFP $)$ transgenic line, and $\operatorname{Tg}\left(\operatorname{heg} 1^{\Delta 25} ; f l k 1: e G F P\right)$ transgenic line [10].

\section{Drug treatment}

Zebrafish $\operatorname{Tg}(\mathrm{cmlc2}$ :eGFP) embryos were incubated in egg water until $48 \mathrm{~h}$-post-fertilization (hpf). Four experimental groups were set up as: control group, TFD group, TFD + NXT group and NXT group. Embryos were collected in 6-well plates with 15 embryos per well and three replicates were set for each group. The control group was treated with $0.1 \%$ dimethyl sulfoxide (DMSO) for $48 \mathrm{~h}$; the NXT group was treated with NXT at a concentration of $600 \mathrm{mg} / \mathrm{L}$ for $48 \mathrm{~h}$; the TFD group was treated with $15 \mu \mathrm{M}$ TFD (Solarbio, China) for $48 \mathrm{~h}$ to obtain zebrafish cardiomyopathy model [9]. For TFD + NXT group, the embryos were treated with $15 \mu \mathrm{M}$ TFD for $48 \mathrm{~h}$ first, followed by NXT treatment for another $24 \mathrm{~h}$.
To optimize the concentrations of NXT, the following doses of NXT were used, 200, 300, 400, 500, 600, 700, 800, 900 and $1000 \mathrm{mg} / \mathrm{L}$. For heg1 mutants rescue experiment, heg1 mutant embryos at $48 \mathrm{hpf}$ were collected and placed into 6-well plates. To optimize the concentrations of NXT, 500, 1000, 1500, 2000, 2200, 2400, 2500, 2600, and $2800 \mathrm{mg} / \mathrm{L}$ of NXT were used to treat heg1 mutant embryos for $24 \mathrm{~h}$. The recovery rate of heart rate and blood flow were recorded at the end of the treatment, 45 embryos per group were used for statistics analysis.

\section{Cardiac morphology analysis}

After treatment, embryos were collected at $96 \mathrm{hpf}$ for phenotype analysis. Zebrafish embryos were mounted in $5 \%$ methylcellulose. Images were obtained using a Nikon SMZ25 stereomicroscope (Nikon, Japan) for morphology observation. Photographs were analyzed using NISElements BR software (Nikon, Japan) to determine the distance between sinus venosus and bulbus arteriosus (SV-BA) and pericardial area per field of view.

\section{Heart rate and blood flow rate statistics}

The heart beats of embryos per unit of time was counted with a stopwatch and a counter, expressed as beats per minute (BPM). Video imaging of the movement of red blood cells (RBCs) in the posterior cardiac veins (PVCs) of 96 hpf zebrafish embryos was acquired. DanioScope software (Noldus, Netherland) was used to analyze the movement ratio of RBCs based on changes in pixel density, and the results were expressed as the ratio of blood flow pixel values to surrounding tissue.

\section{Heart isolation of zebrafish embryos}

Zebrafish embryonic heart dissection was carried out according to the procedure adapted from Lombardo [24]. Briefly, 200-300 Tg(cmlc2:eGFP) zebrafish embryos at $96 \mathrm{hpf}$ from four groups were collected respectively, and placed in $1.5 \mathrm{~mL}$ tube on ice. $1 \mathrm{~mL} \mathrm{L-15/10 \%} \mathrm{FBS}$ medium was added. The embryos pericardial cavity was destructed using a $5 \mathrm{~mL}$ syringe with a 20-gauge needle. GFP-positive hearts were separated manually under fluorescence microscope and placed in L-15/10\% FBS medium for further study.

\section{RNA-seq and bioinformatic analysis}

After zebrafish embryonic hearts were isolated, total RNA was extracted from four group samples of zebrafish embryonic hearts using Trizol (Life Technologies, USA), including control group, NXT group, TFD group, and TFD + NXT group, respectively [25]. Libraries were constructed by using NEBNext Ultra ${ }^{\mathrm{TM}}$ RNA Library Prep Kit for Illumina (NEB, USA) and sequenced by Illumina novaseq 6000 platform (NovoTech, China). The clean 
reads were mapped to reference genome (Danio rerio: NCBI_GRCz11). Differential expressed genes (DGE) analysis of the two groups was performed using the DESeq2 $\mathrm{R}$ package (1.10.1). The $p$ values were adjusted using the Benjamini and Hochberg's approach for controlling the false discovery rate. Genes with an adjusted $p$ value $<0.001$ and $\mid \log _{2}$ (Fold change) $\mid \geq 2$ were considered as differentially expressed.

The DEGs were subjected to enrichment analyses of Gene Ontology (GO) and KEGG pathways analysis. GO terms and KEGG analysis with corrected $p$ value $<0.05$ were considered as significantly enriched.

\section{Real-time quantitative PCR (qRT-PCR)}

For qRT-PCR analysis, total RNA was extracted from whole embryos of zebrafish in each group using TRIzol reagent (Life Technologies, USA). The cDNA was synthesized using the SuperScriptIII (Invitrogen, USA). qRT-PCR detection was conducted by the SYBR FAST Universal qPCR kit (KAPA, Germany) and ViiA 7 RealTime PCR System (ABI, USA). The reaction system was as follows: $95{ }^{\circ} \mathrm{C}$ pre-denaturation for $3 \mathrm{~min} ; 95^{\circ} \mathrm{C}$ for $5 \mathrm{~s}, 60^{\circ} \mathrm{C}$ for $30 \mathrm{~s}, 40$ cycles. The quantified values were generated from the average results of three independent experiments with the triplicate PCR runs [21]. Primer sequences are listed (Additional file 1: Table S1).

\section{Chemical analysis of NXT by UPLC}

The composition of NXT was identified using Ultra Performance Liquid Chromatography (UPLC) method. The mixed chemical standards, including mulberroside A (MulA), hydroxysafflor yellow A (HSYA), amygdalin (AMY), paeoniflorin (PF), ferulic acid (FA), calycosin 7-O-glucoside (CG), rosmarinic acid (RA), salvianolic acid $B($ Sal B), calycosin (CAL), formononetin (FN) and tanshinone IIA (Tan IIA) were bought from National Instisutes for Food and Drug Control (China). The separation was achieved using a WATERS ACQUITY UPLC BEH C18 column $(2.1 \mathrm{~mm} \times 100 \mathrm{~mm}, 1.7 \mu \mathrm{m})$, with a mobile phase consisting of $0.2 \%$ formic acid (phase A) and acetonitrile (phase B) at a flow rate of $0.2 \mathrm{~mL} / \mathrm{min}$ at a temperature of $35{ }^{\circ} \mathrm{C}$. UV detection at $254 \mathrm{~nm}$ was applied. The UPLC fingerprint of NXT was recorded and analyzed by comparing with the references.

Control embryos or TFD-induced embryos were treated with PF $(10 \mu \mathrm{M})$ or Sal B $(10 \mu \mathrm{M})$ or PF:SalB (1:1) mixture, respectively. All phenotypical and qRT-PCR analysis were described as above.

\section{Statistical analysis}

Each experiment was conducted at least three times. Data analysis was performed using Graph Pad Prism 5.0 software. All data were presented as means \pm SD. Statistical evaluation was performed by one-way ANOVA analysis. $p<0.05$ was considered as statistically significant.

\section{Results}

NXT restored TFD-induced cardiomyopathy in zebrafish

To investigate the myocardial protective effect of NXT on cardiomyopathy, we used $15 \mu \mathrm{M}$ TFD to establish a zebrafish cardiomyopathy model. As shown in Fig. 1, compared with control group, zebrafish in the TFD treated group exhibited severe cardiac malformations, including pericardial edema (Fig. 1A, red dash line), venous congestion (Fig. 1A, red arrow), dramatically blood flow reduction (Fig. 1B), increased pericardial area (Fig. 1D), slow heart rate (Fig. 1E), as well as abnormally increased SV-BA distance (Fig. 1F). The zebrafish cardiomyocyte specific transgenic reporter line $T g(\mathrm{cmlc2}: e G F P)$, when treated with TFD, the embryo atrium was dilated, with abnormal atrial (A) and ventricular (V) morphology (Fig. 1G). This is similar to the pathophysiology of dilated cardiomyopathy (DCM).

After treatment with different concentrations of NXT (200-1000 $\mathrm{mg} / \mathrm{L})$, the cardiomyopathy phenotype induced by TFD was restored to varying degrees. The optimal recovery concentration was $600 \mathrm{mg} / \mathrm{L}$ for further experiments (Fig. 1C). Compared with the TFD group, the TFD + NXT group embryos exhibited a 65\% decrease in the enlarged pericardial area (Fig. 1A, D), a significant recovery of blood flow (Fig. 1B), a 44.3\% reduction in abnormal increased SV-BA distance (Fig. 1F), and a close to normal atrium and ventricle morphology (Fig. 1G). The heart rate of TFD group was reduced to $80 \pm 5 \mathrm{BPM}$ compare with the heart rate of $175 \pm 5 \mathrm{BPM}$ in the control group, and the heart rate was restored to $132 \pm 5$ BPM in TFD + NXT group (Fig. 1E). Therefore, NXT could significantly restore the phenotype of TFD induced cardiomyopathy in zebrafish.

\section{Transcriptome analysis of zebrafish cardiomyopathy embryo hearts treated with NXT}

In order to identify the molecular targets of NXT in the treatment of cardiomyopathy, $\mathrm{Tg}(\mathrm{cmlc2}$ :eGFP) zebrafish embryonic hearts at $96 \mathrm{hpf}$ were isolated from each group, including control, TFD, TFD + NXT, and NXT, respectively (Fig. 2A, B). Total RNA was extracted from zebrafish embryonic hearts followed by RNA-Seq analysis. There were 17,771 transcripts expressed in the four transcriptome groups (Fig. 2C). The expression patterns of screened genes were subjected to cluster analysis as shown in Fig. 2D.

After quality filtering, a total of 961 differentially expressed genes (DEGs) was identified in TFD and TFD + NXT groups $\left(\mid \log _{2}(\right.$ FoldChange $) \mid \geq 2$, $p<0.001)$. Among these genes, 441 genes were 
A

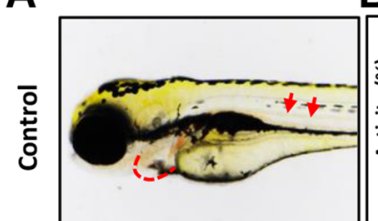

B
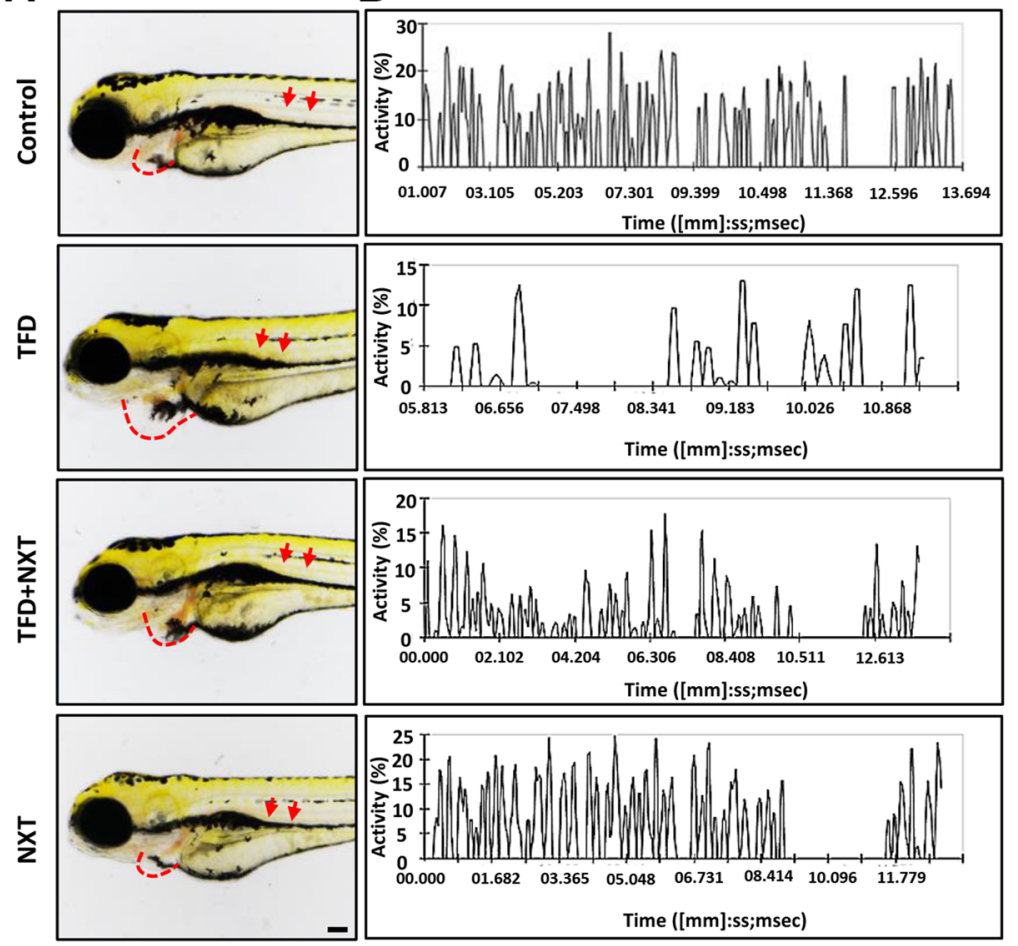

G

Control

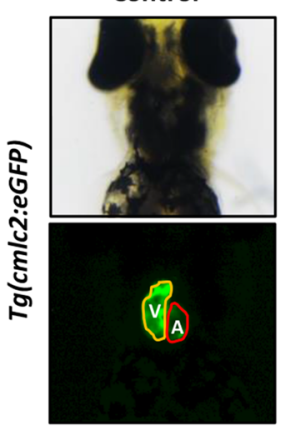

TFD

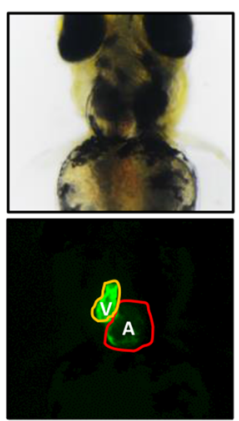

TFD+NXT

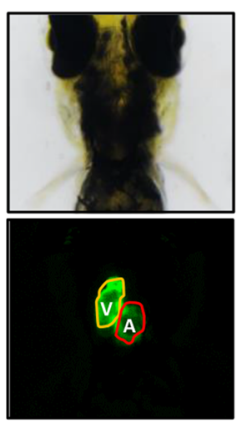

NXT

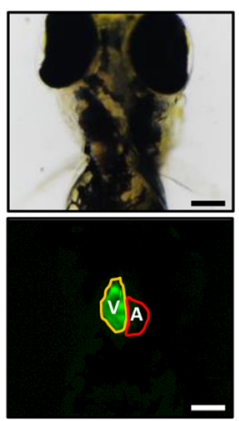

C

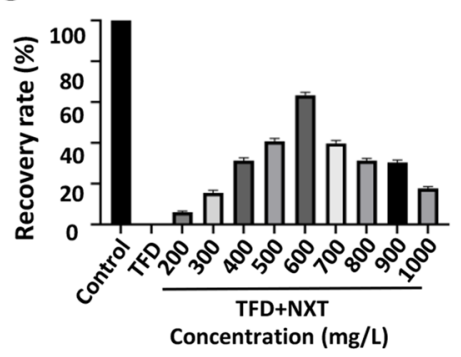

D

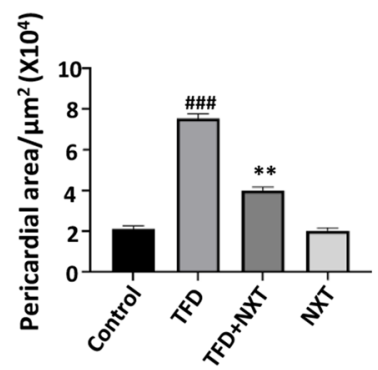

$\mathbf{E}$

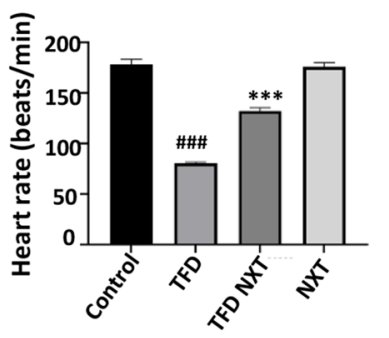

$\mathbf{F}$

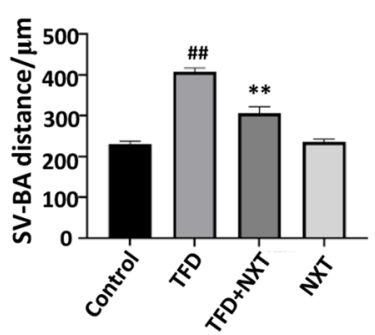

Fig. 1 NXT treatment restored TFD-induced cardiomyopathy in zebrafish embryos. A Lateral view of four groups of zebrafish embryos at $4 \mathrm{dpf}$. TFD treated group showing pericardial edema (red dashed line) and slowed blood flow (red arrows). B Blood flow motion ratios of four groups of zebrafish embryos based on pixel density changes in RBCs. C Optimal recovery concentration of NXT. D Pericardial area of four groups of zebrafish larvae ( $n=15$ embryos/group). E Heart rates of four groups of zebrafish larvae ( $n=15$ embryos/group) F SV-BA distance of the four groups of zebrafish larvae ( $n=15$ embryos/group). G The heart morphology of four groups embryos at $96 \mathrm{hpf}$ delineated by $\mathrm{Tg}(\mathrm{cm} / \mathrm{c2}: \mathrm{GFP})$ (ventral view), Note the enlarged ventricles in the TFD treatment group ( $V$ ventricle; $A$ atria). Data are represented mean \pm standard deviation (SD) from three independent experiments, ${ }^{\# \#} p<0.01,{ }^{\# \#} p<0.001$ vs control group; ${ }^{* *} p<0.01,{ }^{* * *} p<0.001$ vs TFD-induced group (Student's t test)

upregulated and 520 genes were downregulated (Fig. 3A, Additional file 2: Table S2). Not surprisingly, a number of genes highly associated with cardiac development were altered, including those involved in cardiac muscle contraction (up-regulated: 27; downregulated: 12) and heart development (up-regulated: 34; down-regulated: 6) (Fig. 3B).
In order to explore whether this change was related to specific pathways or biological process, we carried out Gene ontology (GO) functional enrichment analysis and KEGG pathway enrichment analysis (Additional file 3: Table S3). It was found that the upregulated genes were mainly enriched in cardiovascular system development, including heart development, blood circulation, 
A

Zebrafish embryonic heart dissection

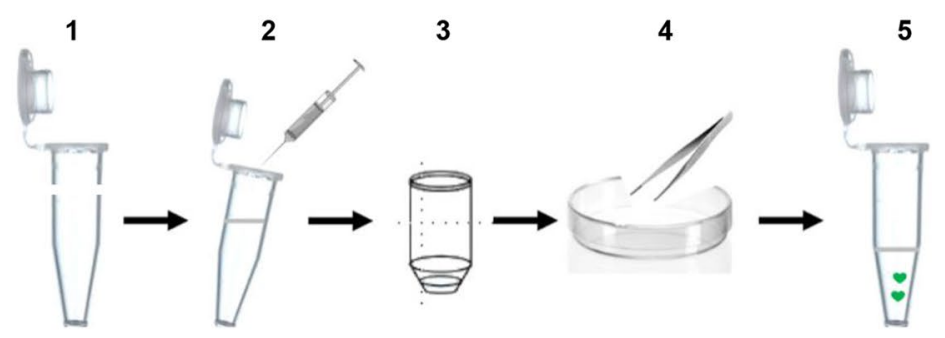

B
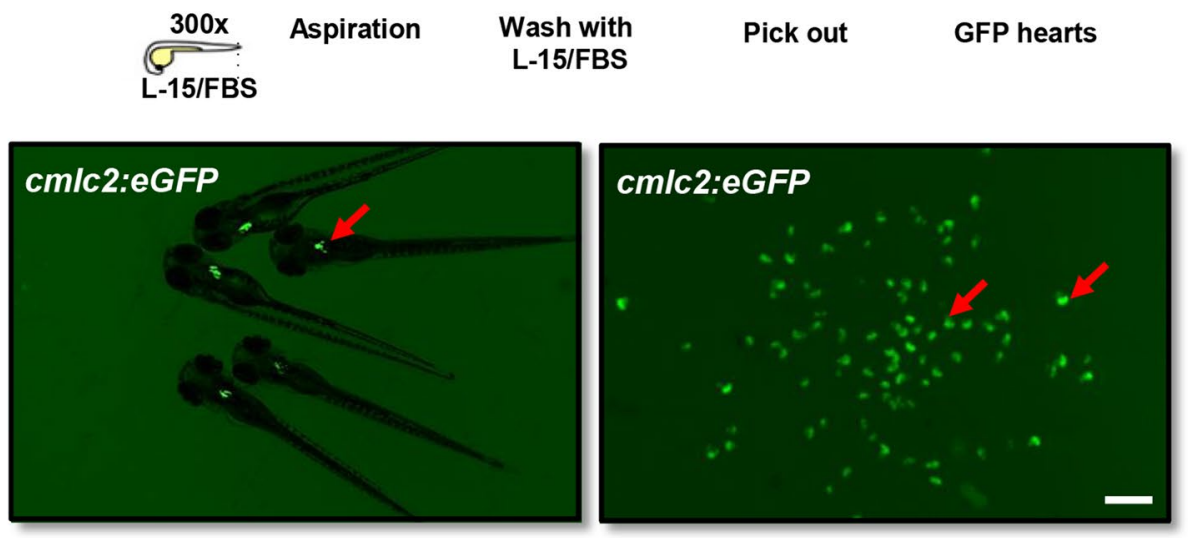

C

D
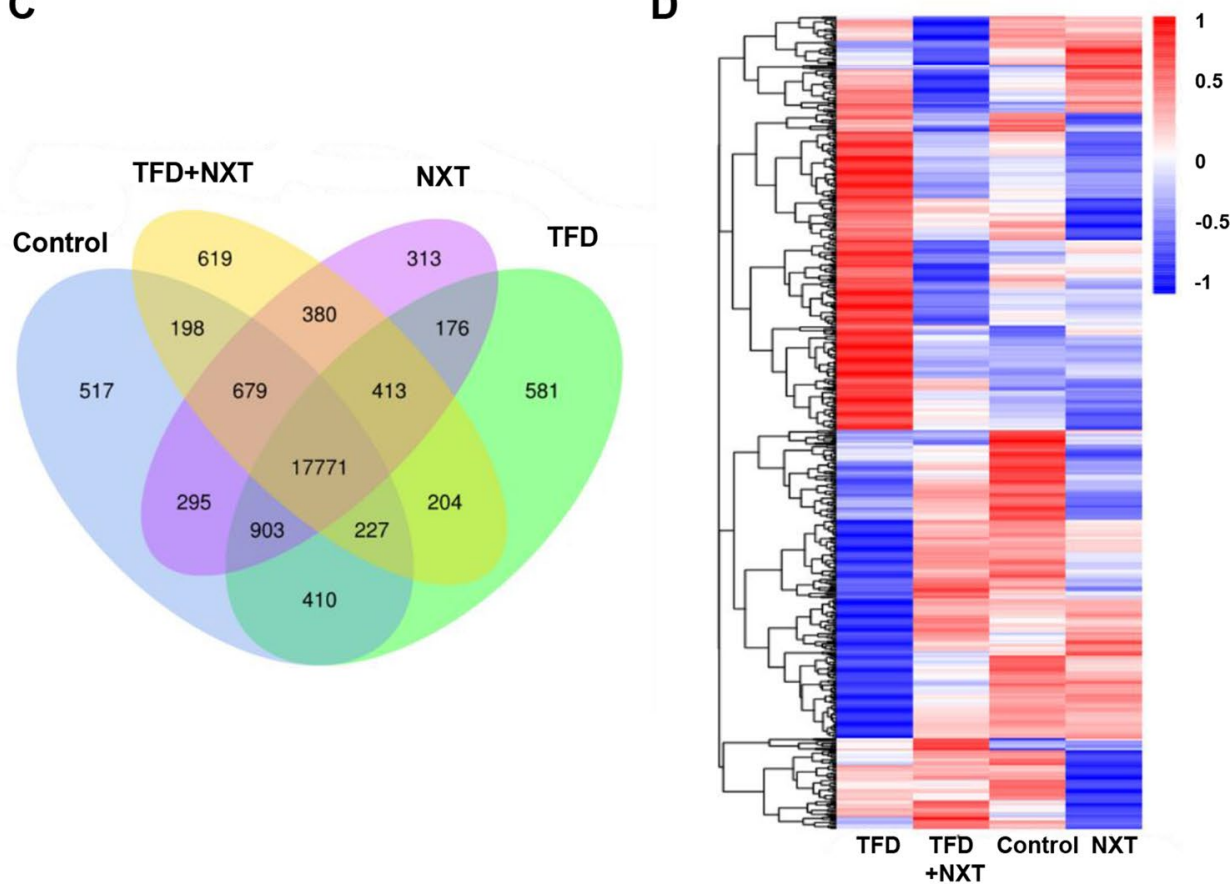

Fig. 2 Transcriptome analysis of differentially expressed genes in NXT treated cardiomyopathy zebrafish hearts. A A schematic overview of heart isolation in zebrafish embryos. B The isolated cardiac tissues from $\mathrm{Tg}(\mathrm{cm} / \mathrm{cz}$ :eGFP) transgenic fish embryos at 96 hpf. C Venn diagram analysis and D Heat map representation showing DGEs in control, TFD, TFD + NXT and NXT group 
A

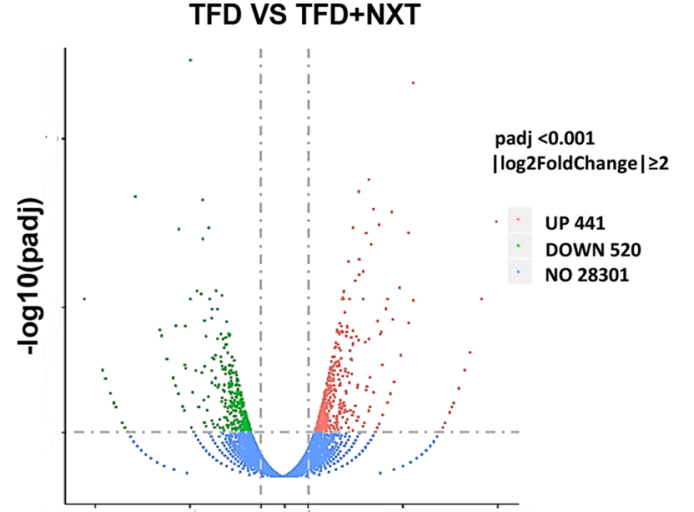

C

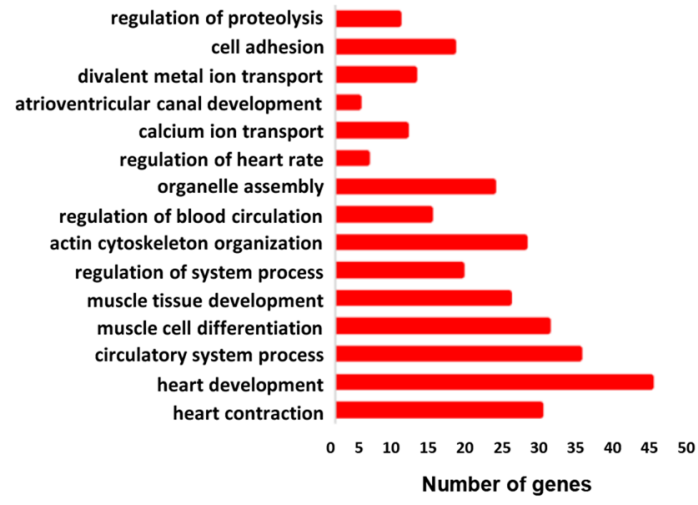

E

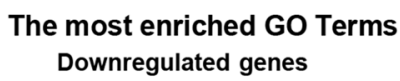

Downregulated genes

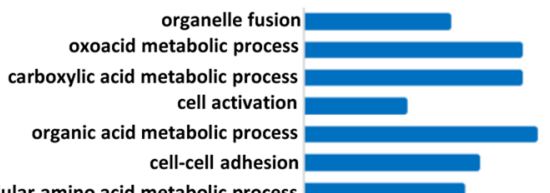

cellular amino acid metabolic process

aromatic amino acid family metabolic process

secretion by cell

regulation of immune system process

negative regulation of molecular function regulation of proteolysis protein activation cascade

regulation of endopeptidase activity

negative regulation of proteolysis

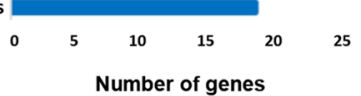

B

\section{Cardiac muscle contraction Heart development}
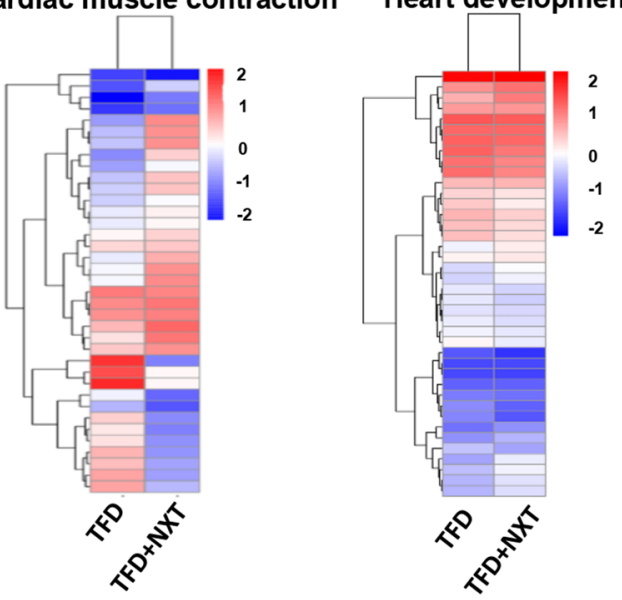

D

\section{Pathway enrichment}

Upregulated genes

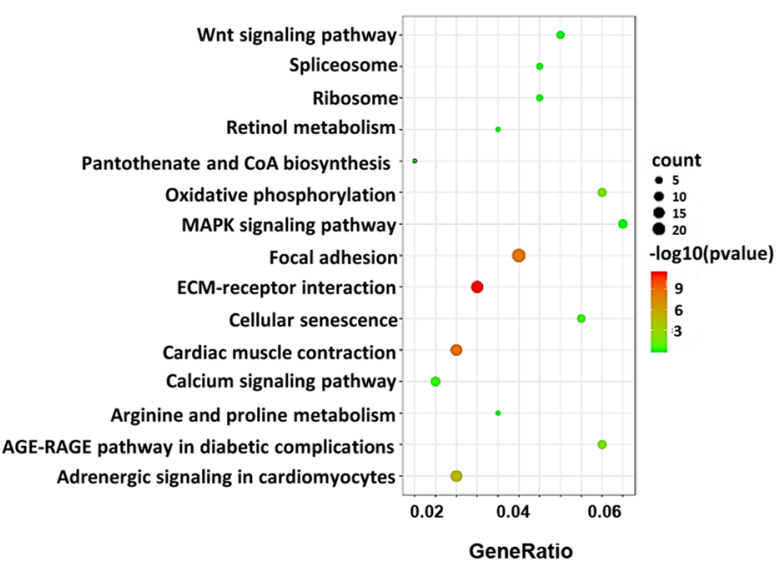

$\mathbf{F}$

Pathway enrichment Downregulated genes

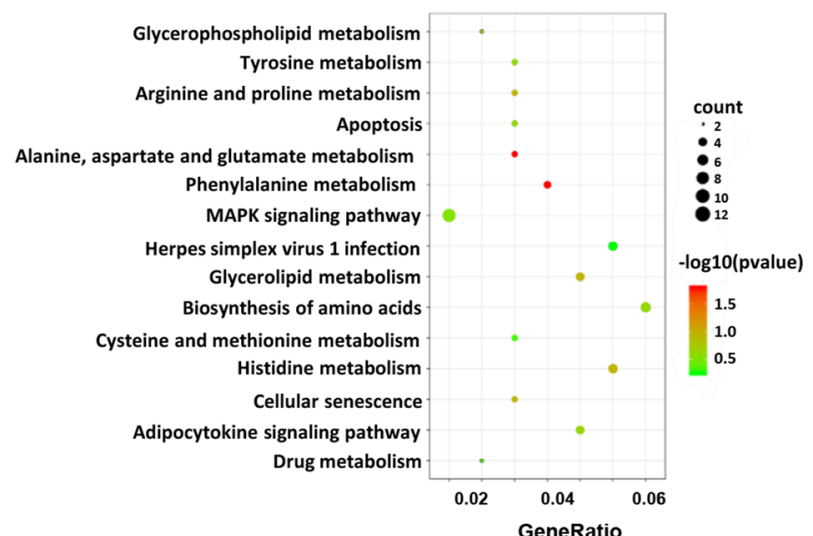

Fig. 3 Bioinformatics analysis between TFD and TFD + NXT groups. A Volcano plots showing a total of 961 DGEs in TFD and TFD + NXT groups. B A heat-map showed that genes involved in cardiac muscle contraction and heart development. C GO term analysis and D KEGG pathway analysis for upregulate DEGs in TFD + NXT zebrafish embryo hearts compared to TFD group. E GO term analysis and F KEGG pathway analysis for upregulate DEGs in TFD + NXT zebrafish embryo hearts compared to TFD group. The size of the dot indicates the number of genes enriched in individual item. The color of the dot represents the $p$ value 
and cardiac muscle cell and tissue development (Fig. 3C); the downregulated genes were enriched in regulation of proteolysis, endopeptidase activity, immune system process, and the metabolic process of oxoacid, carboxylic acid, and organic acid (Fig. 3E). KEGG analysis also revealed that the upregulated genes were mainly in cardiac muscle contraction and ECM-receptor interaction pathways (Fig. 3D); the downregulated genes were mainly in MAPK signaling pathway and metabolism pathways of glycerophospholipid and amino acid (Fig. 3F). This finding suggested that the restorative effect of NXT on zebrafish cardiomyopathy might be achieved by influencing key myocardial signaling pathways and the biological process of cardiac development.

\section{NXT alleviated zebrafish cardiomyopathy by regulating of myocardial and cardiovascular development related genes}

Previous studies of human genetics have shown that there are multiple genes related to dilated cardiomyopathy (DCM) [26-29]. Based on RNA-Seq data obtained here from zebrafish heart, we summarized 30 DCM related genes homologous to human in zebrafish (Table 1), including cardiac actin, cardiac troponin, myosin heavy chain, myosin-binding protein, and so on. Among those genes relevant to DCM, myocardial-related genes (tnnc2, myhz1.1, tpm4b, myh6, myh7, myh7ba, scl8a1a, atp1b2a and cacng $3 b)$ and cardiovascular development-related genes (vegfc, nkx2.7, heg1, ccm2, ccm2l, klf2a, kdrl, id4 and fosl2) were revealed to be changed in NXT + TFD treated embryos compared with TFD-induced cardiomyopathy embryos as indicated by RNA-seq analysis. The expression pattern of the two group genes was clustered (Fig. 4A, B). qRT-PCR validation in zebrafish whole embryos further exhibited that the abnormal expression of these genes in TFD embryos was all significantly recovered to normal expression levels in varying degrees upon addition of NXT when compared with the expressions of these genes in control (Fig. 4C, D). This indicated that the therapeutic effect of NXT on DCM might be achieved by regulating genes related to myocardial and cardiovascular development.

\section{HEG1 signaling was one of targets of NXT in the treatment of cardiomyopathy}

HEG1 (heart development protein with EGF-like domains 1) is a transmembrane receptor expressed in the endocardium, which mainly regulates adhesion between heart and vascular cells and regulates myocardial growth [30]. Studies in mice identified HEG1-CCM signaling as a critical regulator of cardiovascular organ formation and integrity [31]. In the RNA-seq data, heg1 and $\mathrm{ccm}$ genes were screened as genes related to cardiovascular development in the zebrafish cardiomyopathy embryo heart when treated with NXT. It was suggested that HEG1-CCM signaling might be one of molecular targets of NXT in the treatment of cardiomyopathy.

To further verify heg1 signaling, heg1 ${ }^{\Delta 25}$ mutant, a congenital zebrafish cardiomyopathy model generated in our lab preciously [10] was treated with NXT. Similar to what observed in TFD-induced cardiomyopathy embryos, heg $1^{\triangle 25}$ mutant embryo at $4 \mathrm{dpf}$ was exhibited obvious pericardial swelling (Fig. 5A, D, red dotted line), blood flow stagnation and thromboembolism caused by cardiac pumping dysfunction (Fig. 5A, B), decreased heart rate (Fig. 5E), and abnormal increased SV-BA distance (Fig. 5F) when compared with control embryos. NXT was used to treat $h e g 1^{\Delta 25}$ mutant at $2 \mathrm{dpf}$ to find the optimal concentration for recovery $(500 \mathrm{mg} / \mathrm{L}-2800 \mathrm{mg} / \mathrm{L})$, and the phenotype was observed at $4 \mathrm{dpf}$ (Fig. 5C). Under the treatment of $2500 \mathrm{mg} / \mathrm{L} \mathrm{NXT}$, those cardiovascular phenotypes of heg $1^{\Delta 25}$ mutant were restored at various degrees, including pericardial area, blood flow, heart rate and SV-BA distance (Fig. 5A, B, D-F). To better observe the cardiovascular phenotype restoration of NXT in heg1 ${ }^{\triangle 25}$ mutant, two heg1 transgenic lines were used, $\operatorname{Tg}\left(h e g 1^{\triangle 25}\right.$; cmlc2:eGFP) and $\operatorname{Tg}\left(\right.$ heg1 ${ }^{\triangle 25}$; fik1:eGFP) [10]. When treated with NXT, the enlarged heart chambers, especially atria, and coagulated blood vessels in heg1 ${ }^{\triangle 25}$ mutant were significantly restored (Fig. 6A, B). Furthermore, the expression of myocardial tissue-specific markers, such as $c m l c 2, m y h 6, m y h 7$, and the vascular markers such as vegfc, scl, flt4 were abnormally decreased in heg $1^{\Delta 25}$ mutant, which were restored to normal or near normal levels after treatment with NXT. These data suggested that the cardiovascular malformations of heg $1^{\triangle 25}$ mutant could be restored under NXT treatment, and HEG1 signaling might be one of important molecular targets of NXT in the treatment of cardiomyopathy.

\section{Identification of NXT components by UPLC}

As shown in Fig. 7A, there were seven major components in NXT identified as HSYA, PF, FA, CG, RA, Sal B, and Tan IIA, respectively. Among them, PF has vasodilatory and inhibitory effects on myocardial infarction and atherosclerosis [32]; Sal B can promote blood vessel growth, improve blood flow as well as prevent atherosclerosis [33]. The myocardial protective activity of PF and Sal B was investigated in TFD-induced zebrafish cardiomyopathy model by phenotypic examination (Additional file 4: Fig. S1) and qRT-PCR analysis (Fig. 7B-D). It indicated that PF and Sal B could significantly recovery cardiomyopathy phenotype (Additional file 4: Fig. S1), and restore abnormal expression of the vascular markers (Fig. 7C) and myocardial tissue-specific markers (Fig. 7D), as well as HEG1-CCM signaling (Fig. 7B). 
Table 1 Zebrafish Homologues associated with human DCM genes based on RNA-seq data

\begin{tabular}{|c|c|c|c|c|c|}
\hline Human DCM-associated gene & $\begin{array}{l}\text { Zebrafish } \\
\text { orthologue }\end{array}$ & Gene ID & Cardiomyopathy subtype(s) & $\log _{2}$ (FoldChange) & padj \\
\hline \multirow[t]{2}{*}{ ACTC1 (cardiac actin) } & actcla & ENSDARG00000042535 & DCM, HCM, LVNC & 2.605409266 & 2.23E-07 \\
\hline & $a c t c 1 b$ & ENSDARG00000076126 & DCM, HCM, LVNC & 3.204639486 & 7.79E-10 \\
\hline MYH6 (myosin heavy chain 6) & myh6 & ENSDARG00000090637 & $\mathrm{DCM}, \mathrm{HCM}, \mathrm{CHD}$ & 2.966691137 & 4.27E-09 \\
\hline \multirow[t]{5}{*}{ MYH7 (myosin heavy chain 7) } & myh7 & ENSDARG00000079564 & DCM, HCM, LVNC & 2.62564815 & $1.82 \mathrm{E}-07$ \\
\hline & myh7ba & ENSDARG00000076075 & DCM, HCM, LVNC & 3.181888154 & $5.38 \mathrm{E}-10$ \\
\hline & myh7bb & ENSDARG00000035322 & DCM, HCM, LVNC & 2.377845495 & $3.79 \mathrm{E}-06$ \\
\hline & myh7l & ENSDARG00000079782 & DCM, HCM & 2.9740424 & 3.10E-09 \\
\hline & myhz1.1 & ENSDARG00000067990 & $\mathrm{DCM}, \mathrm{HCM}$ & 2.59151567 & 8.45E-05 \\
\hline \multirow[t]{2}{*}{ TNNC (cardiac troponin C) } & tnncla & ENSDARG00000011400 & HCM, LVNC & 2.529089309 & $6.54 \mathrm{E}-07$ \\
\hline & tnnc $1 b$ & ENSDARG00000037539 & HCM, LVNC & 2.626641531 & 3.45E-07 \\
\hline \multirow[t]{3}{*}{ TNNT (cardiac troponin T) } & tnnt1 & ENSDARG00000037954 & DCM, HCM, LVNC & 3.661572878 & $1.30 \mathrm{E}-05$ \\
\hline & tnnt2a & ENSDARG00000020610 & DCM, HCM, LVNC & 2.345399238 & $3.72 \mathrm{E}-06$ \\
\hline & tnnt $2 b$ & ENSDARG00000100694 & DCM, HCM, LVNC & 3.182087004 & 4.43E-04 \\
\hline \multirow[t]{3}{*}{ TNNI (cardiac troponin I) } & tnni4a & ENSDARG00000099870 & DCM & 4.604088258 & $7.20 \mathrm{E}-16$ \\
\hline & tnni1b & ENSDARG00000052708 & DCM, HCM & 2.918318845 & 7.07E-09 \\
\hline & tnni3k & ENSDARG00000086933 & DCM, HCM & 2.40317011 & $5.28 \mathrm{E}-04$ \\
\hline \multirow[t]{2}{*}{ TTN (titin) } & $\operatorname{ttn} .1$ & ENSDARG00000000563 & DCM, HCM, ARVC & 2.614507859 & 2.41E-07 \\
\hline & $\operatorname{ttn} .2$ & ENSDARG00000028213 & DCM, HCM, ARVC & 3.388962093 & 2.25E-11 \\
\hline MYBPC3 (myosin-binding protein C) & mybpc3 & ENSDARG00000011615 & DCM, HCM & 3.171024301 & $3.74 \mathrm{E}-10$ \\
\hline NEXN (nexilin) & nexn & ENSDARG00000057317 & DCM, HCM & 2.13189099 & 2.36E-04 \\
\hline \multirow[t]{2}{*}{ NKX (NK2 homeobox) } & $\mathrm{nkx} 2.5$ & ENSDARG00000018004 & $\mathrm{DCM}, \mathrm{HCM}$ & 2.318274388 & 4.34E-05 \\
\hline & $\mathrm{nkx} 2.7$ & ENSDARG00000021232 & DCM & 2.074287056 & 4.63E-04 \\
\hline $\begin{array}{l}\text { HEG1 (heart development protein with EGF } \\
\text { like domains 1) }\end{array}$ & heg1 & ENSDARG00000018441 & DCM & 3.092539 & 4.49E-09 \\
\hline TBX20 (T-box 20) & $\mathrm{tb} \times 20$ & ENSDARG00000005150 & DCM & 1.87288 & 4.85E-04 \\
\hline CCM2L (CCM2 like scaffold protein) & $\mathrm{ccm} 2 \mathrm{l}$ & ENSDARG00000063089 & DCM & 2.074250424 & $2.49 \mathrm{E}-04$ \\
\hline SLC8A1 (solute carrier family 8 member A1) & slc8a1a & ENSDARG00000013422 & $\mathrm{DCM}, \mathrm{HCM}$ & 3.344777218 & 7.19E-11 \\
\hline LAMA2 (laminin, a2) & lama2 & ENSDARG00000099390 & DCM & 1.937049252 & $3.66 \mathrm{E}-03$ \\
\hline \multirow[t]{2}{*}{ TPM4 (tropomyosin 4) } & tpm4a & ENSDARG00000023963 & $\mathrm{DCM}, \mathrm{HCM}$ & 2.275421133 & 7.24E-06 \\
\hline & tpm4b & ENSDARG00000019128 & $\mathrm{DCM}, \mathrm{HCM}$ & 1.94540804 & $2.02 \mathrm{E}-04$ \\
\hline ACTN2 (actinin, a2) & $a c t n 2 b$ & ENSDARG00000071090 & $\mathrm{DCM}, \mathrm{HCM}$ & 2.537681759 & 8.35E-07 \\
\hline CSRP3 (cysteine and glycine-rich protein 3) & csrp3 & ENSDARG00000101706 & $\mathrm{DCM}, \mathrm{HCM}$ & 5.082307638 & $5.42 \mathrm{E}-19$ \\
\hline EYA4 (eyes absent homolog 4) & eya4 & ENSDARG00000012397 & DCM & 1.763847085 & $2.92 \mathrm{E}-03$ \\
\hline
\end{tabular}

The fold change and adjusted $p$ value were calculated by the R package DESeq in zebrafish embryonic heart treated with TFD over that treated with TFD + NXT, with $\mid \log _{2}($ FoldChange) $\mid>1$ and padj $<0.01$

DCM dilated cardiomyopathy; HCM hypertrophic cardiomyopathy; LVNC left ventricular noncompaction; RCM restrictive cardiomyopathy; $A R V C$ arrhythmogenic right ventricular cardiomyopathy; CPVT cathecolaminergic polymorphic ventricular tachycardia

\section{Discussion}

Cardiomyopathy is a type of progressive heart disease in which the heart is abnormally enlarged, thickened, or rigid. It causes cardiac dysfunction due to damage to the myocardium and defective myocardial contractility. As a result, the heart becomes weaker and is less able to pump blood through the body, often causing heart failure and abnormal heart rhythms. Here, by using a TFDinduced zebrafish cardiomyopathy model, we were able to demonstrate that NXT could restore the reduced heart rate, the enlarged heart chambers, the reduced cardiac systolic function and the cardiac pumping dysfunction in zebrafish, indicating that NXT significantly improved cardiac dysfunction and had a good therapeutic effect on cardiomyopathy.

NXT has been approved by the Sino Food and Drug Administration (SFDA) (Z20025001) for clinical treatment of CVD. NXT had been shown to prevent atherosclerosis by improving blood lipid levels and restoring intestinal micro-ecological imbalance [20]. In a rat model of coronary heart disease with qi deficiency and blood stasis, NXT was found to regulate blood lipid 


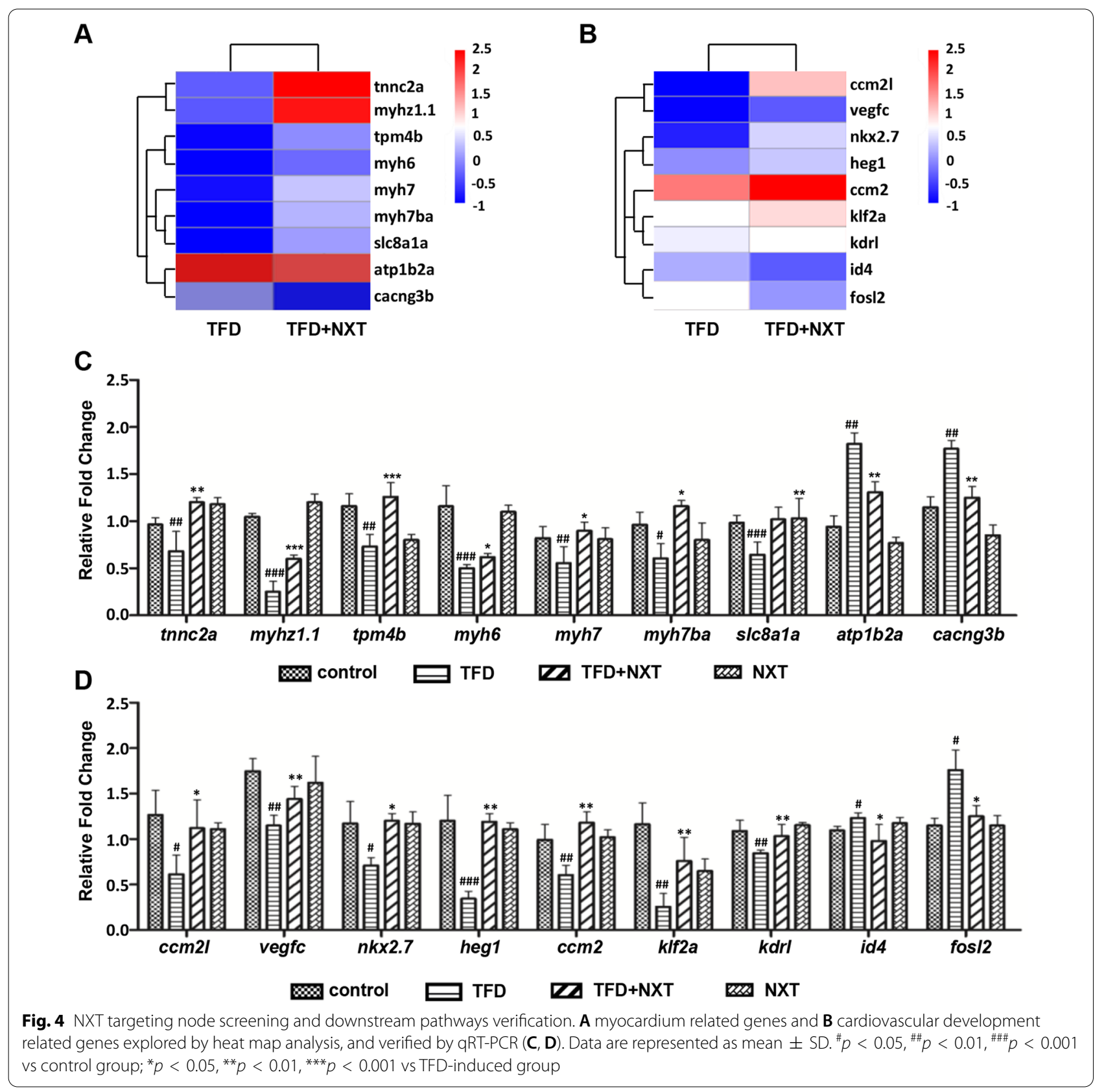

levels, enhance antioxidant capacity, inhibit vascular inflammation and reduce myocardial damage, so as to inhibit the occurrence of coronary heart disease. The protective effect of NXT on myocardium in the treatment of coronary artery disease was mainly achieved by reducing the level of creatine kinase-MB [33]. A metaanalysis of randomized trials was performed in 2018, including 11 studies $(\mathrm{N}=1141)$ showing that NXT is an effective and safe therapy option for patients with cerebral infarction and carotid atherosclerosis [34]. However, the underlying molecular mechanism of NXT in the treatment of cardiomyopathy is still not well elucidated. In this study, zebrafish embryonic hearts were isolated from TFD treated group and TFD + NXT treated group, respectively, for transcriptome and bioinformatics analyses. A total of 961 DEGs was screened between TFD and TFD + NXT treated zebrafish hearts by RNA-seq analysis, including 441 up-regulated genes and 520 down-regulated genes. Furthermore, by comparing $30 \mathrm{DCM}$ related genes homologous to human in zebrafish hearts based on RNA-Seq data, heg1 was identified as one of the important targets via 
A
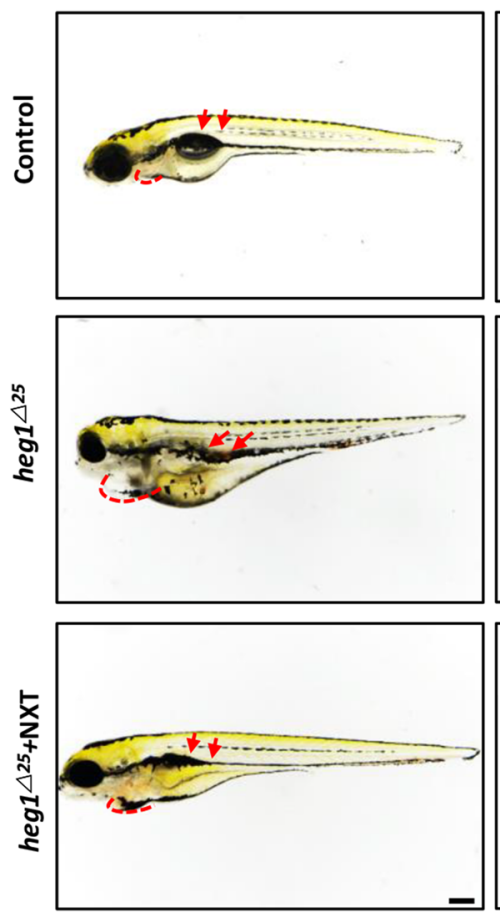

C

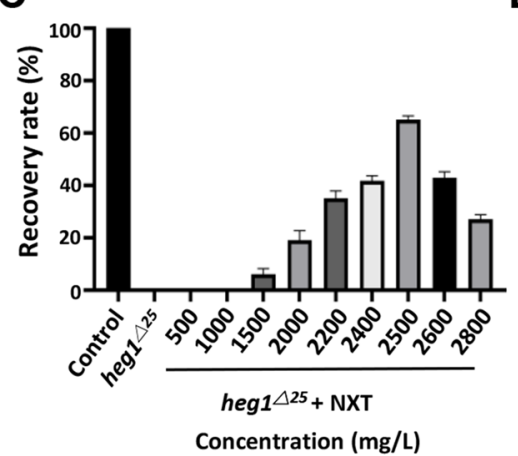

B
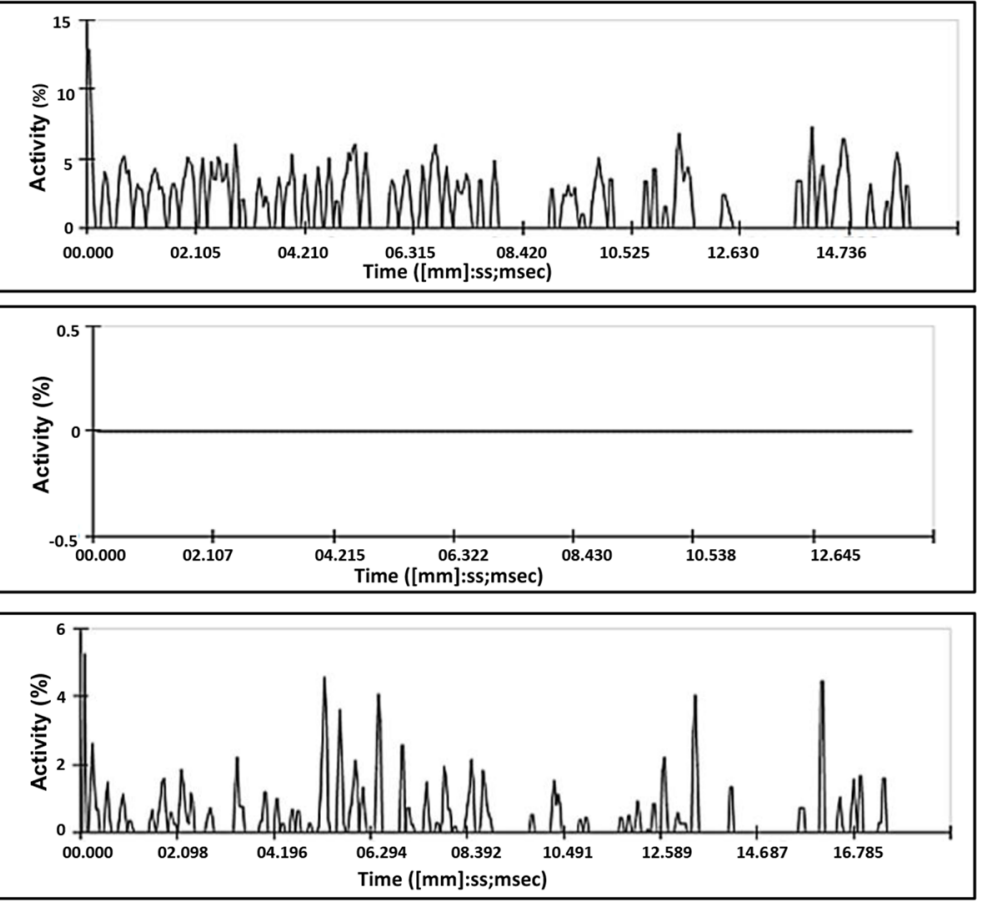

D

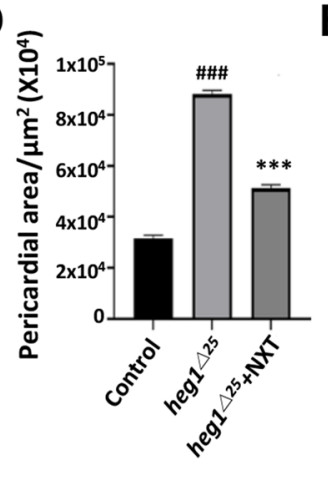

$\mathbf{E}$

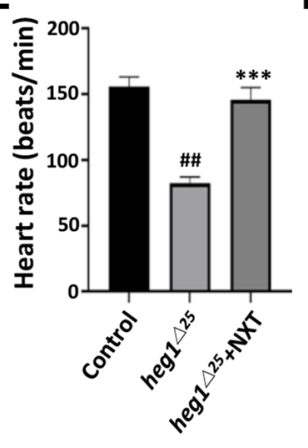

$\mathbf{F}$

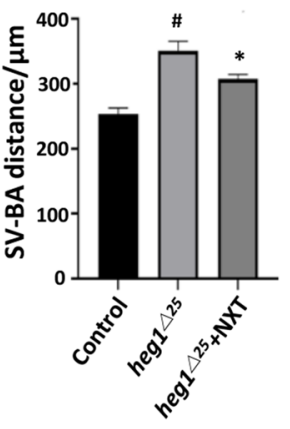

Fig. 5 NXT restored cardiovascular malformation in heg ${ }^{\Delta 25}$ mutant. A Lateral views of zebrafish embryos at 96 hpf. heg ${ }^{\Delta 25}$ mutant embryos showing pericardial edema (red dashed line) and venous congestion (red arrows). B Blood flow motion ratios of three groups of zebrafish embryos based on RBCs pixel density changes. C Optimal recovery concentration of NXT. D Pericardial area of the three groups of zebrafish larvae ( $\mathrm{n}=15$ embryos/group). $\mathbf{E}$ Heart rates of three groups of zebrafish larvae ( $\mathrm{n}=15$ embryos/group). F SV-BA distance of three groups of zebrafish larvae ( $n=15$ embryos/group). ${ }^{\#} p<0.05,{ }^{\# \#} p<0.01,{ }^{\# \#} p<0.001$ vs control group; ${ }^{*} p<0.05,{ }^{* * *} p<0.001$ vs heg ${ }^{\Delta 25}$ mutant group

which NXT could restore cardiomyopathy in zebrafish through HEG1-CCM signaling.

As a receptor, HEG1 usually binds to CCM1 and acts on heart and vascular development together with CCM1, CCM2 and PDCD10. The HEG1-CCM signaling pathway is crucial for cardiovascular development in zebrafish and mice [35]. In zebrafish, mutants $c c m 1$ (santa/san), ccm2 (valentine/vtn), and heg1 exhibited severe cardiac malformations and hemorrhagic arrest [30, 36]. In addition, knockdown of $c c m 2 l$ (ccm2-like), an enhancer in the HEG1-CCM pathway, also caused dilatation of the atria and reduced or absent blood circulation in zebrafish [37]. RNA-seq analysis in this study revealed that heg1ccm signaling was down-regulated in TFD-induced cardiomyopathy zebrafish embryonic hearts. However, the abnormal down-expressions of heg1, ccm2, and $c \mathrm{~cm} 2 l$ in TFD-induced cardiomyopathy could be restored by NXT treatment confirmed by qRT-PCR validation. To further verify heg1 signaling regulated by NXT, heg1 ${ }^{\triangle 25}$ mutant, a congenital DCM zebrafish model was applied 


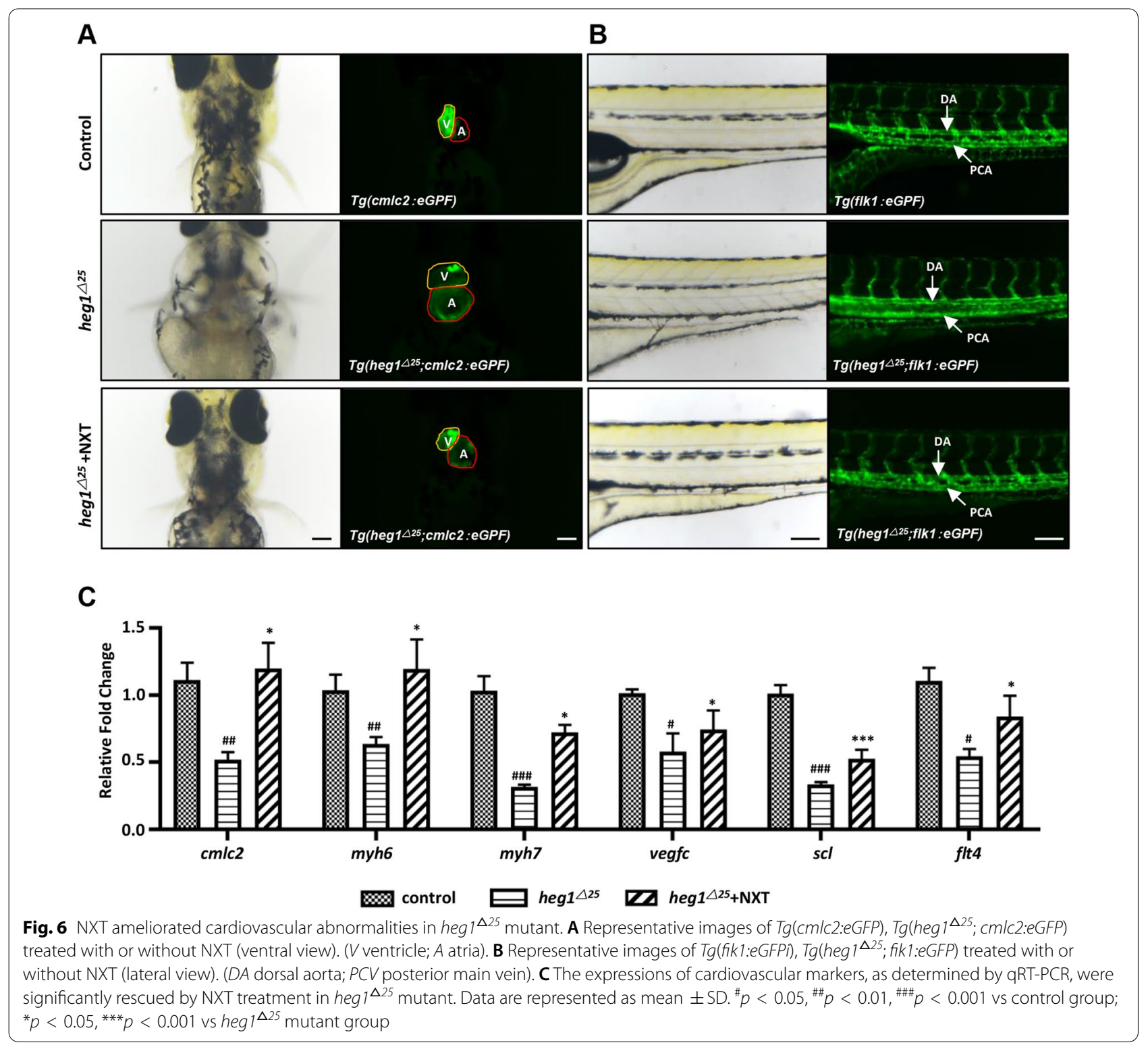

to evaluate the therapeutic effect of NXT. The treatment of NXT on heg1 ${ }^{\Delta 25}$ mutant not only significantly rescued the cardiomyopathy phenotypes, including the enlarged hearts and the stagnation of blood flow caused by cardiac pumping dysfunction, but also restored the abnormal expression of myocardial tissue-specific markers and vascular markers. These results suggested that NXT is a promising TCM compound for the treatment of cardiomyopathy.

Studies have shown that DCM is the result of multiple pathological mechanisms leading to cardiac dilation and associated cardiac dysfunction, with the changes in cardiac contractility and cardiac morphology accompanied by alterations in myocardial gene expression
[38]. Through RNA-Seq screening of cardiomyopathy zebrafish embryonic heart treated with or without NXT, genes involved in myocardial contraction and myocardial tissue development were enriched. Among them, myosin heavy chain proteins $(\mathrm{MYH})$ reflect the development of myofibrils and regulate cardiac contraction and cardiac physiological characteristics. There are nine MYH homologous genes in zebrafish [28]. Previous studies have shown that atrial myosin heavy chain (myh6/amhc) is specifically expressed in zebrafish atria, while ventricular myosin heavy chain $(m y h 7 / v m h c)$ is predominantly expressed in zebrafish ventricle [39]. Mutations of myh6 and $m y h 7$ in zebrafish are known to be associated with cardiomyopathy. The loss of myh6 function will impair 
A

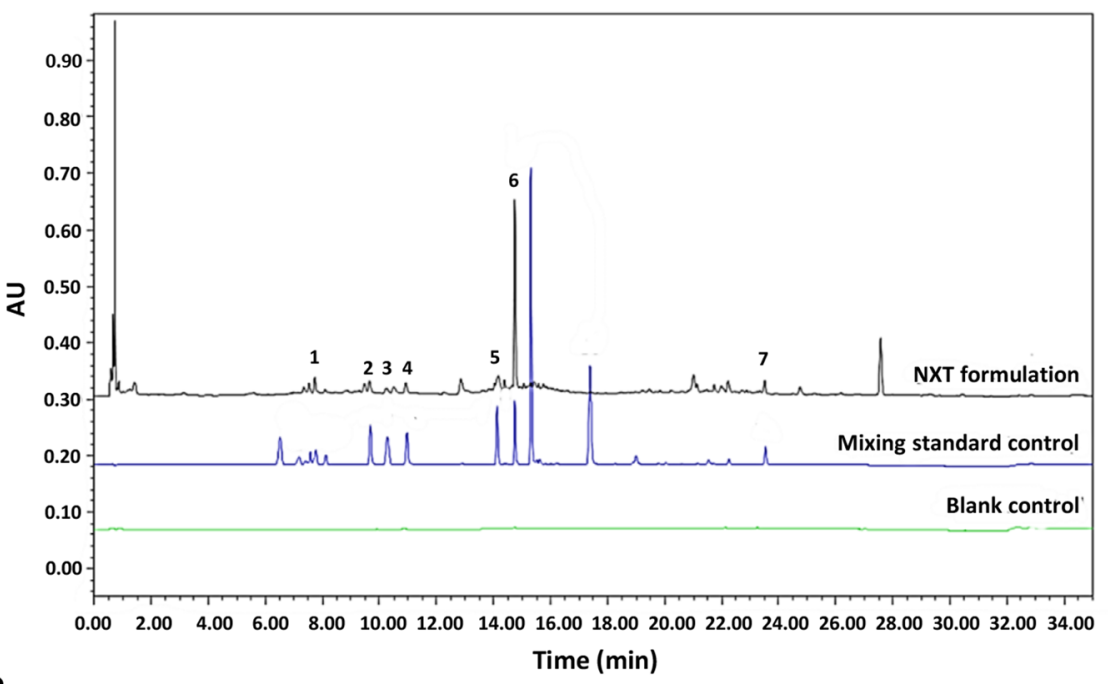

B
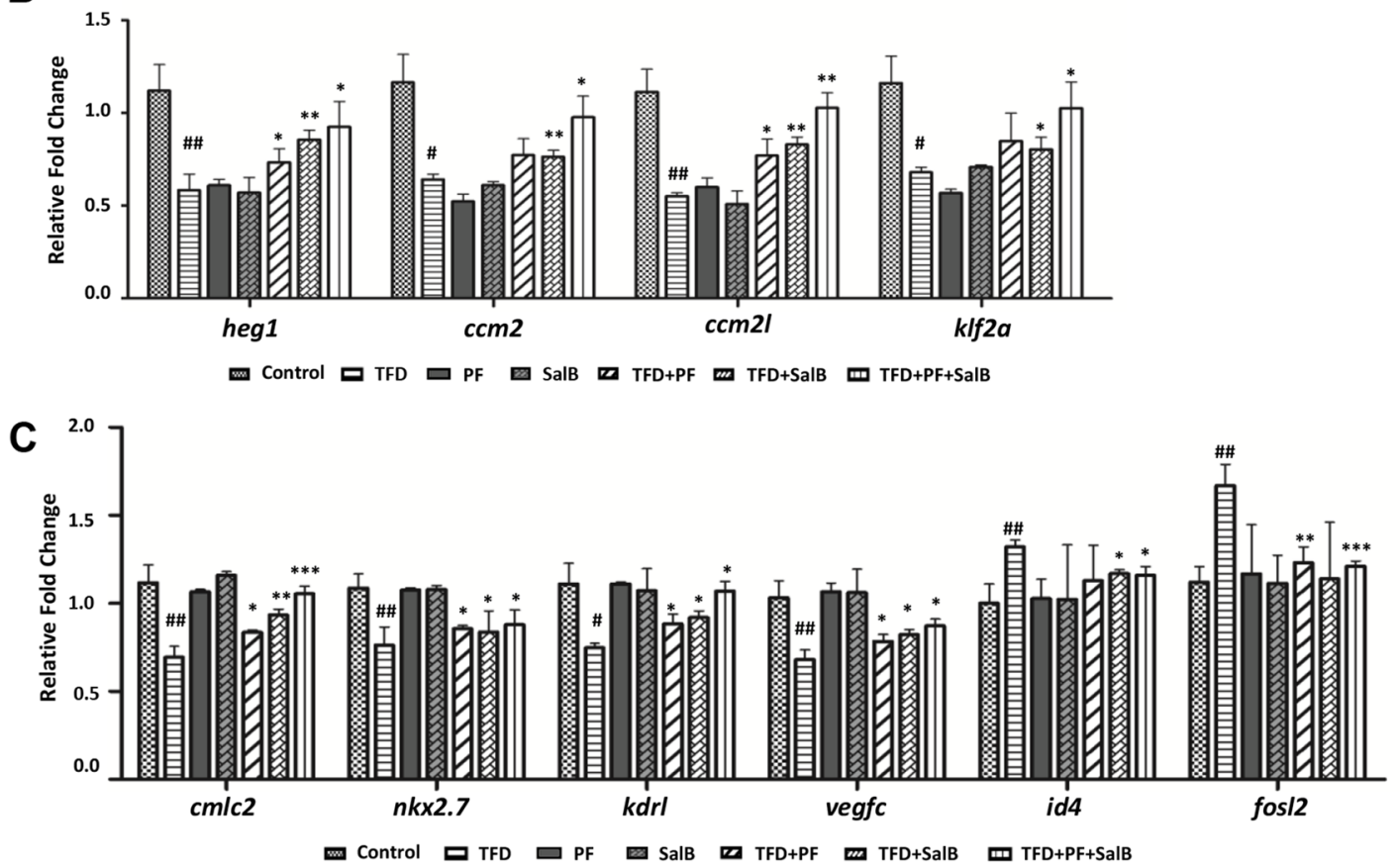

D

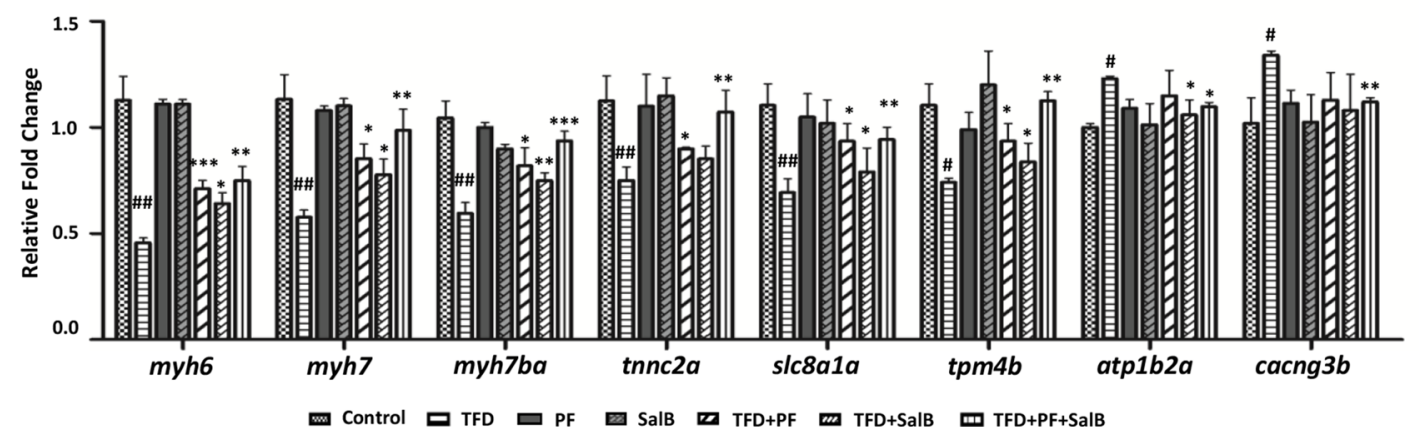

Fig. 7 UPLC analysis of NXT combined with bioassays. A The fingerprints of the NXT. 1: HSYA, 2: PF, 3: FA, 4: CG, 5: RA, 6: Sal B, 7: Tan IIA. B HEG1-CCM signaling, $\mathbf{C}$ cardiovascular-related genes, D myocardial-related genes verified by qRT-PCR. Data are represented as mean \pm SD. ${ }^{\#} p<0.05$, \#\# $p<$ $0.01,{ }^{\# \# \#} p<0.001$ vs control group; ${ }^{*} p<0.05,{ }^{* *} p<0.01,{ }^{* * *} p<0.001$ vs TFD-induced group 
atrial contractility and atrial muscle tubercle assembly, but will not affect ventricular contraction; while the loss of myh7 function will lead to excessive formation of myocytes and reduction of myogenic fibers in the ventricle $[28,40]$. Therefore, myh6 and myh7 are important molecular biomarkers of zebrafish heart remodeling, and their expression directly affects the development and functional regulation of zebrafish heart. In the process of regulating myocardial contraction, cardiac troponin $\mathrm{T}$ (TNNT), cardiac troponin I (TNNI) and cardiac troponin $\mathrm{C}$ (TNNC) form troponin complexes, which are the preferred biomarkers of myocardial necrosis and provide a calcium-sensitive switch for myocardial contraction. TNNI serves to inhibit the interaction of actin and tropomyosin and to activate $\mathrm{Ca}^{2+}$ sensitivity through its interaction with TNNC. During contraction, calcium binds to TNNC, resulting in a conformational change that enhances the binding of TNNC to TNNI and regulates cardiac contraction [41]. In our study, the transcriptional expression of myh6, myh7, myh7ba, and tnnc2 genes were significantly reduced in zebrafish embryos with cardiomyopathy, which directly affected myocardial structure formation, contraction and calcium ion regulation of zebrafish. Whereas NXT treatment could significantly restore the abnormal decreased expression of these genes, suggesting that NXT could promote the development of myofibril and the assembly of myosin, improve calcium ion sensitivity, and thus regulate myocardial contraction, not only in zebrafish, but also in humans.

\section{Conclusion}

Through TFD-induced zebrafish cardiomyopathy model and the heg1 mutant of the congenital zebrafish cardiomyopathy model, we proved that NXT had a good therapeutic effect on cardiomyopathy, and could restore myocardial injury and cardiac dysfunction. Transcriptome and bioinformatics analyses further revealed that NXT could restore cardiomyopathy in zebrafish through HEG1-CCM signaling, which might be one of molecular pathological mechanisms of NXT in the treatment of cardiovascular diseases. In addition, PF and Sal B could be considered as major bioactive components of NXT for myocardial protection in cardiomyopathy.

\footnotetext{
Abbreviations

NXT: Naoxintong; TCM: Traditional Chinese Medicine; TFD: Terfenadine; CVD: Cardiovascular diseases; HCM: Hypertrophic cardiomyopathy; DCM: Dilated cardiomyopathy; RCM: Restrictive cardiomyopathy; ARVC: Arrhythmogenic right ventricular cardiomyopathy; SV-BA: Sinus venosus and bulbus arteriosus; I/R: Ischemia/reperfusion; RBCs: Red blood cells; PVCs: Posterior cardiac veins; qRT-PCR: Real-time quantitative PCR; UPLC: Ultra performance liquid chromatography; MulA: Mulberroside A; HSYA: Hydroxysafflor yellow A; AMY: Amygdalin; PF: Paeoniflorin; FA: Ferulic acid; CG: Calycosin 7-O-glucoside; RA: Rosmarinic acid; Sal B: Salvianolic acid B; CAL: Calycosin; FN: Formononetin; Tan IIA: Tanshinone IIA; GO: Gene ontology; HEG1: Heart development protein
}

with EGF-like domains 1; MYH: Myosin heavy chain proteins; TNNT: Cardiac troponin T; TNNI: Cardiac troponin I; TNNC: Cardiac troponin C.

\section{Supplementary Information}

The online version contains supplementary material available at https://doi. org/10.1186/s13020-021-00532-0.

\begin{abstract}
Additional file 1: Figure S1. Restoration of TFD-induced zebrafish embryonic cardiomyopathy by PF and Sal B, two main components of NXT. A Lateral view of seven groups of zebrafish embryos at $4 \mathrm{dpf}$. TFD treated group showing pericardial edema (red dashed line) and slowed blood flow (red arrows). B Blood flow motion ratios of seven groups of zebrafish embryos based on pixel density changes in RBCs. $\mathbf{C}$ Heart rates of seven groups of zebrafish larvae ( $n=15$ embryos/group). D Pericardial area of seven groups of zebrafish larvae ( $n=15$ embryos/group). E SV-BA distance of the seven groups of zebrafish larvae ( $n=15$ embryos/group). Data are represented mean \pm standard deviation (SD) from three independent experiments, ${ }^{\# \# \#} p<0.001$ vs control group; ${ }^{* *} p<0.01$, ${ }^{* * *} p<0.001$ vs TFD-induced group.

Additional file 2: Table S1. List of all primer sequences.

Additional file 3: Table S2. DGEs of TFD+NXT vs. TFD.

Additional file 4: Table S3. GO and KEGG analysis of DGEs of TFD+NXT vs. TFD.
\end{abstract}

\section{Acknowledgements}

Not applicable.

\section{Authors' contributions}

Conceptualization, JT and $\mathrm{MH}$; funding acquisition, JT and FL; formal analysis, $\mathrm{MH}$ and $\mathrm{PL}$; methodology, $\mathrm{MH}$ and $\mathrm{SL}$; data curation, $\mathrm{PL}$ and $\mathrm{HL}$; visualization, $Z W$ and $Z L$; resources: $Y S$; supervision: $F L$; writing original draft preparation, $\mathrm{MH}$; writing review and editing, JT. All authors read and approved the final manuscript.

\section{Funding}

This work was supported by Shaanxi key industry innovation chain (Group) Foundation in social development field (2019ZDLSF02-05); Opening Foundation of Key Laboratory of Resource Biology and Biotechnology in Western China, Ministry of Education (ZSK2018010); National standardization project of TCM (ZYBZH-C-QIN-45); Shaanxi Science and technology overall planning and innovation project (2016KTTSSF01-02); Shaanxi key R\&D program (2017ZDXM-SF-012).

Availability of data and materials

The research data generated from this study is included within the article.

\section{Declarations}

Ethics approval and consent to participate

This study was approved by the Experimental Animal Management and Ethics Committee of Northwest University, the ethical code was NWU-AWC-20190103Z.

Consent for publication

Not applicable.

Competing interests

The authors declare that they have no competing interests.

\section{Author details}

${ }^{1}$ Western China Zebrafish Research Center for Human Diseases and Drug Screening, The College of Life Sciences, Northwest University, Xi'an 710069, China. ${ }^{2}$ Key Laboratory of Resource Biology and Biotechnology in Western China, Ministry of Education, School of Medicine, Northwest University, Xi'an 710069, China. ${ }^{3}$ Shaanxi Buchang Pharmaceutical Co. Ltd., Xi'an 710075, 
China. ${ }^{4}$ Shaanxi Institute of International Trade and Commence, Xi'an 712046, China.

Received: 20 July 2021 Accepted: 1 November 2021

Published online: 14 November 2021

\section{References}

1. Burke MA, Cook SA, Seidman JG, Seidman CE. Clinical and mechanistic insights into the genetics of cardiomyopathy. J Am Coll Cardiol. 2016;68(25):2871-86.

2. Imai M, Rastogi S, Gupta RC, Mishra S, Sharov VG, Stanley WC, Mika Y, et al. Therapy with cardiac contractility modulation electrical signals improves left ventricular function and remodeling in dogs with chronic heart failure. J Am Coll Cardiol. 2007:49(21):2120-8.

3. Brieler J, Breeden MA, Tucker J. Cardiomyopathy: an overview. Am Fam Physician. 2017;96(10):640-6.

4. Yu J, Zeng C, Wang Y. Epigenetics in dilated cardiomyopathy. Curr Opin Cardiol. 2019;34(3):260-9.

5. Bakkers J. Zebrafish as a model to study cardiac development and human cardiac disease. Cardiovasc Res. 2011;91(2):279-88.

6. Genge CE, Lin E, Lee L, Sheng X, Rayani K, Gunawan M, Stevens CM, et al. The zebrafish heart as a model of mammalian cardiac function. Rev Physiol Biochem Pharmacol. 2016;171:99-136.

7. Kesavan G, Chekuru A, Machate A, Brand M. CRISPR/Cas9-mediated zebrafish knock-in as a novel strategy to study midbrain-hindbrain boundary development. Front Neuroanat. 2017;11:52.

8. Asnani A, Peterson RT. The zebrafish as a tool to identify novel therapies for human cardiovascular disease. Dis Model Mech. 2014;7(7):763-7.

9. Gu G, Na Y, Chung H, Seok SH, Lee HY. Zebrafish larvae model of dilated cardiomyopathy induced by terfenadine. Korean Circ J. 2017;47(6):960-9.

10. Lu S, Hu M, Wang Z, Liu H, Kou Y, Lyu Z, Tian J. Generation and application of the zebrafish heg1 mutant as a cardiovascular disease model. Biomolecules. 2020;10(11):1542.

11. Zhu L, Chen Z, Han K, Zhao Y, Li Y, Li D, Wang X, et al. Correlation between mitochondrial dysfunction, cardiovascular diseases, and traditional chinese medicine. Evid Based Complement Alternat Med. 2020;2020:2902136.

12. Wan J, Wan $H$, Yang $R$, Wan $H$, Yang J, He Y, Zhou H. Protective effect of Danhong Injection combined with Naoxintong Capsule on cerebral ischemiareperfusion injury in rats. J Ethnopharmacol. 2018;211:348-57.

13. Li J, Bai Y, Bai Y, Zhu R, Liu W, Cao J, An M, Tan Z, Chang YX. Pharmacokinetics of caffeic acid, ferulic acid, formononetin, cryptotanshinone, and tanshinone IIA after oral administration of Naoxintong capsule in rat by HPLC-MS/MS. Evid Based Complement Alternat Med. 2017;2017:9057238.

14. Han J, Tan H, Duan Y, Chen Y, Zhu Y, Zhao B, Wang Y, Yang X. The cardioprotective properties and the involved mechanisms of Naoxintong capsule. Pharmacol Res. 2019;141:409-17.

15. Wang Z, Liu P, Hu M, Lu S, Lyu Z, Kou Y, Sun Y, et al. Naoxintong restores ischemia injury and inhibits thrombosis via COX2-VEGF/NFkB signaling. J Ethnopharmacol. 2021;270:113809.

16. Lv P, Tong $X$, Peng Q, Liu Y, Jin H, Liu R, Sun W, et al. Treatment with the herbal medicine, Naoxintong improves the protective effect of high-density lipoproteins on endothelial function in patients with type 2 diabetes. Mol Med Rep. 2016;13(3):2007-16.

17. Wang H, Zhong WJ, Huang MW, Wu XY, Chen H. Efficacy of dual antiplatelet therapy combined with Naoxintong capsules (see text) following coronary microembolization induced by homologous microthrombi in rats. Chin J Integr Med. 2011;17(12):917-24.

18. Wang $Y$, Yan $X, M i ~ S, ~ L i ~ Z$, Wang Y, Zhu H, Sun $X$, et al. Naoxintong attenuates Ischaemia/reperfusion Injury through inhibiting NLRP3 inflammasome activation. J Cell Mol Med. 2017;21(1):4-12.

19. Xu H, Jin J, Chen L, Li C, Xu Q, Shi J, Zhao B, et al. Naoxintong/PPARa signaling inhibits $\mathrm{H} 9 \mathrm{c} 2$ cell apoptosis and autophagy in response to oxidative stress. Evid Based Complement Alternat Med. 2016;2016:4370381.

20. Zhang WJ, Su WW, Li PB, Rao HY, Lin QW, Zeng X, Chen TB, et al. Naoxintong capsule inhibits the development of cardiovascular pathological changes in bama minipig through improving gut microbiota. Front Pharmacol. 2019;10:1128.
21. Tian J, Shao J, Liu C, Hou HY, Chou CW, Shboul M, Li GQ, et al. Deficiency of Irp4 in zebrafish and human LRP4 mutation induce aberrant activation of Jagged-Notch signaling in fin and limb development. Cell Mol Life Sci. 2019;76(1):163-78.

22. Huang CJ, Tu CT, Hsiao CD, Hsieh FJ, Tsai HJ. Germ-line transmission of a myocardium-specific GFP transgene reveals critical regulatory elements in the cardiac myosin light chain 2 promoter of zebrafish. Dev Dyn. 2003;228(1):30-40.

23. Jin SW, Beis D, Mitchell T, Chen JN, Stainier DY. Cellular and molecular analyses of vascular tube and lumen formation in zebrafish. Development. 2005;132(23):5199-209.

24. Lombardo VA, Otten C, Abdelilah-Seyfried S. Large-scale zebrafish embryonic heart dissection for transcriptional analysis. J Vis Exp. 2015;95:52087.

25. Xiao D, Wang H, Hao L, Guo X, Ma X, Qian Y, Chen H, Ma J, et al. The roles of SMYD4 in epigenetic regulation of cardiac development in zebrafish. PLOS Genet. 2018;14(8):e1007578.

26. Hershberger RE, Hedges DJ, Morales A. Dilated cardiomyopathy: the complexity of a diverse genetic architecture. Nat Rev Cardiol. 2013;10(9):531-47.

27. McNally EM, Mestroni L. Dilated cardiomyopathy: genetic determinants and mechanisms. Circ Res. 2017;121(7):731-48.

28. Shih YH, Zhang Y, Ding Y, Ross CA, Li H, Olson TM, Xu X. Cardiac transcriptome and dilated cardiomyopathy genes in zebrafish. Circ Cardiovasc Genet. 2015;8(2):261-9.

29. Wilsbacher LD. Clinical implications of the genetic architecture of dilated cardiomyopathy. Curr Cardiol Rep. 2020;22(12):170.

30. Mably JD, Mohideen MA, Burns CG, Chen JN, Fishman MC. heart of glass regulates the concentric growth of the heart in zebrafish. Curr Biol. 2003;13(24):2138-47.

31. Kleaveland B, Zheng X, Liu JJ, Blum Y, Tung JJ, Zou Z, Sweeney SM, et al. Regulation of cardiovascular development and integrity by the heart of glass-cerebral cavernous malformation protein pathway. Nat Med. 2009;15(2):169-76.

32. Hu H, Zhu Q, Su J, Wu Y, Zhu Y, Wang Y, Fang H, et al. Effects of an enriched extract of paeoniflorin, a monoterpene glycoside used in chinese herbal medicine, on cholesterol metabolism in a hyperlipidemic rat model. Med Sci Monit. 2017:23:3412-27.

33. Li CL, Liu B, Wang ZY, Xie F, Qiao W, Cheng J, Kuang JY, et al. Salvianolic acid $B$ improves myocardial function in diabetic cardiomyopathy by suppressing IGFBP3. J Mol Cell Cardiol. 2020;139:98-112.

34. Liang $Q$, Cai Y, Chen R, Chen W, Chen L, Xiao Y. The effect of Naoxintong capsule in the treatment of patients with cerebral infarction and carotid atherosclerosis: a systematic review and meta-analysis of randomized trials. Evid Based Complement Alternat Med. 2018;2018:5892306.

35. Zheng X, Riant F, Bergametti F, Myers CD, Tang AT, Kleaveland B, Pan W, et al. Cerebral cavernous malformations arise independent of the heart of glass receptor. Stroke. 2014;45(5):1505-9.

36. Mably JD, Chuang LP, Serluca FC, Mohideen MA, Chen JN, Fishman MC. Santa and valentine pattern concentric growth of cardiac myocardium in the zebrafish. Development. 2006;133(16):3139-46.

37. Rosen JN, Sogah VM, Ye LY, Mably JD. ccm2-like is required for cardiovascular development as a novel component of the Heg-CCM pathway. Dev Biol. 2013;376(1):74-85.

38. Bollen IAE, Schuldt M, Harakalova M, Vink A, Asselbergs FW, Pinto JR, Krüger $M$, Kuster DWD, van der Velden J. Genotype-specific pathogenic effects in human dilated cardiomyopathy. J Physiol. 2017;595(14):4677-93.

39. Berdougo E, Coleman H, Lee DH, Stainier DY, Yelon D. Mutation of weak atrium/atrial myosin heavy chain disrupts atrial function and influences ventricular morphogenesis in zebrafish. Development. 2003;130(24):6121-9.

40. Siddique BS, Kinoshita S, Wongkarangkana C, Asakawa S, Watabe S. Evolution and distribution of teleost myomiRNAs: functionally diversified myomiRs in teleosts. Mar Biotechnol. 2016;18(3):436-47.

41. Westfall MV, Albayya FP, Metzger JM. Functional analysis of troponin I regulatory domains in the intact myofilament of adult single cardiac myocytes. J Biol Chem. 1999;274(32):22508-16.

\section{Publisher's Note}

Springer Nature remains neutral with regard to jurisdictional claims in published maps and institutional affiliations. 